\title{
Data Generation and Multi-Modal Analysis for Recorded Operatic Performance
}

\author{
Joshua Neumann \\ University of Florida \\ joshuaoneumann@ufl.edu
}

\begin{abstract}
Commercial recordings of live opera performance are only sporadically available, mostly due to various legal protections held by opera houses. The resulting onsite, archive-only access for them inhibits analysis of the creative process in "live" environments. Based on a technique I developed for generating performance data from copyright protected archival recordings, this paper presents a means of interrogating the creative practice in individual operatic performances and across the corpus of a recorded performance history. My analysis uses "In questa Reggia” from Giacomo Puccini's Turandot as performed at New York's Metropolitan Opera. The first part of my analysis builds on tempo mapping developed by the Centre for the History and Analysis of Recorded Music. Given the natural relationship in which performances of the same work exist, statistical and network analyses of the data extracted from a corpus of performances offer ways to contextualize and understand how performances create a tradition to which and through which they relate to varying degrees.
\end{abstract}

\section{CCS Concepts}

- General and reference Empirical studies - Mathematics of computing Exploratory data analysis - Information systems Music retrieval - Information systems Clustering - Human-centered computing Graph drawings - Applied computing Sound and music computing • Applied computing Performing arts

\section{Keywords}

Opera; Archival recordings; Data generation; Network analysis

\section{INTRODUCTION}

Operatic performance occupies an apparent paradoxical existence in the minds of practitioners and consumers: it should be at once individualistic yet within the bounds of an observer's sense of tradition. Opera is thus an ideal locus for investigating both the creative process from dramatic impulse to onstage musical behavior in individual performances and the corpus of a recorded performance history $[2,7,9]$. As an operatic narrative unfolds in time, the musical pacing with which this happens plays a fundamental role in conveying the drama of its events. This property is especially evident for the works of the late nineteenth and early twentieth century, where tempo rubato is a prominent marker of musical expressivity $[5,11]$.

Permission to make digital or hard copies of all or part of this work for personal or classroom use is granted without fee provided that copies are not made or distributed for profit or commercial advantage and that copies bear this notice and the full citation on the first page. Copyrights for components of this work owned by others than ACM must be honored. Abstracting with credit is permitted. To copy otherwise, or republish, to post on servers or to redistribute to lists, requires prior specific permission and/or a fee. Request permissions from Permissions@acm.org. DLfM '16, August 12 2016, New York, USA (C) 2016 ACM. ISBN 978-1-4503-4751-8/16/08_..\$15.00 DOI: http://dx.doi.org/10.1145/2970044.2970045
Opera's textual nature makes possible the addition of a layer of nuance for understanding and characterizing the dramatic pacing as it results from musical pacing. Also considering the textual narrative of the drama in this context makes it possible to assess better the creative process. This approach builds on tempo mapping techniques developed at the Centre for the Analysis and History of Recorded Music (CHARM) and the Center for Musical Performance as Creative Practice (CMPC) [2]. Furthermore, as each successive performance contributes to the establishment of a tradition, proximal relationships form between it and each of the other performances and points in the resulting tradition. Analyzing this network of relationships reveals information about performances, their relationship to tradition, the nature of tradition itself.

Within Turandot, the titular character's entrance aria, "In questa Reggia," occupies a prominent place in the dramatic sequence of the work and in production design conceptions. This aria recounts the ostensible reasons for Turandot's riddles and brutality - her desire for vengeance on behalf of her raped and murdered ancestress Lou-o-Ling. The recorded corpus of Turandot at the Metropolitan Opera includes performances in two of the productions, one by Cecil Beaton, which ran from the 1960-61 season through the 1974-75 season, and one by Franco Zeffirelli, dating from the 1986-87 season.

In this paper, I demonstrate analysis of the creative process using temporal-textual emphasis and statistical analysis of tempo data in a primarily archival corpus of live opera recordings. Comparing performances' sonic data along with that of the traditions they create reveals characteristics of their relationships to each, the nature of tradition, and, in this instance, the influence of production practice on performance practice.

\section{PARAMETERS AND METHODS}

\subsection{Case Study Parameters}

Table 1 - Singers and Years for "In questa Reggia" at the Metropolitan Opera

\begin{tabular}{|l|l|}
\hline Singer & Performance \# (Conductor - date) \\
\hline Birgit Nilsson & P1 (Leopold Stokowski - 1961) \\
& P2 (Kurt Adler - 1962) \\
& P3 (Fausto Cleva - 1965) \\
& P4 (Zubin Mehta - 1966) \\
& P6 (Kurt Adler - 1970) \\
\hline Marion Lippert & P5 (Mehta - 1969) \\
\hline Elinor Ross & P7 (Gabor Ötvös - Apr. 1974) \\
\hline Ingrid Bjoner & P8 (Alberto Erede - Dec. 1974) \\
\hline Eva Marton & P9 (James Levine - Mar. 1987) \\
& P10 (Levine - Apr. 1987) \\
\hline Ghena Dimitrova & P11 (Nello Santi - 1988) \\
& P15 (Santi - 1996) \\
\hline Gwyneth Jones & P12 (Santi - 1990) \\
& P13 (Santi - 1992) \\
& P14 (Santi - 1995) \\
\hline Adrienne Dugger & P16 (Marco Armiliato - 2003) \\
\hline Andréa Gruber & P17 (Bertrand de Billy - 2005) \\
& P18 (Armiliato - 2007) \\
\hline Maria Guleghina & P19 (Andris Nelsons - 2009 \\
\hline
\end{tabular}


Table 1 lists singers of recorded performances of "In questa Reggia" at the Metropolitan Opera; these nineteen recordings represent this aria's entire accessible corpus at the USA's preeminent opera house. No other opera company has made available as many archival recordings of performances of Turandot on its stage. Access to such recordings, though limited, is access to a substantial recorded performance history.

\subsection{Data Generation Methods}

Both digital (CD and DVD) and analog (cassette or reel tape) recordings of Turandot comprise the available corpus. Some of these items are commercially available through legitimate means while others are only accessible at NYPL. Bootleg recordings are available for all others identified here, and many other recordings in Turandot's performance history, both for sale or via internet streaming or download. This study analyzed only legally accessible recordings. Furthermore, as this aria changes meter between $2 / 4,4 / 8,2 / 8$, and $4 / 4$, automated measure delineations are not possible. However, since each measure contains an even number of eighth notes and not all quarter notes have a discernible subdivision, beat calculation at the level of a quarter note suffices for the current study. Furthermore, these methods are a first foray in this arena and may thus change with continued use and development.

\subsubsection{Commercially available recording data extraction}

For recordings that are commercially available, either in digital form or able to be converted into it, the data generation method uses the same approach as the Mazurka Project [8]. Upon loading the file into Sonic Visualiser, beat entry occurred with reference to a score. Beat markers were moved as necessary, and then the onset detector plugin was applied to refine marker positioning. Tempo-to-following-beat data extraction followed.

\subsubsection{Archive-only available recordings data extraction}

Copyrights prohibit any duplication of recordings not already commercially available, thus alternative means of generating tempo data are necessary. For this process, I adopted principles of aural dictation. First, I listened to a recording and made annotations in a score to reflect places of tempo stasis and tempo modulation. On a second listening, I created a tempo-taptrack using the voice notes recorder on a cellular phone. The next step was to listen to the tap track in one ear and the aria recording in the other, making further notes in the score of passages that were accurate and others that needed adjustment. This refining process repeated several times. Upon loading these audio files into Sonic Visualiser, all tap tracks were compared against each other, and in reference to score annotations, either the most accurate tap track section's beat marker was used, or, in isolated instances, a beat marker was assigned to the average of the closest tap tracks. While obviously imperfect, this is one of the only means available for generating data from copyright protected recordings. Furthermore, any discrepancies that do exist between final beat marker assignment and actual beat placement within the current data set are within hundredths, if not thousandths of a second. The same method of extracting tempo data was then applied to these tap tracks as to recordings with digital copies commercially available.

\subsection{Time Scape Analysis for Opera}

Using Time Scapes' inherent ability to reveal varying strengths of tempo relationships across a performance is a natural technique for opera, especially since this genre relies upon musical and dramatic pacing to communicate its narrative. This analysis uses the same method as Cook and Sapp applied in the Mazurka Project, and adds a beat scale, textual markers, and a tempo contour graph to the Time Scape $[8,9]$. These additions help emphasize opera's textual nature and reveal textual connections within a performance's time structure. Surface level analysis indicates how deviations from the global tempo have the effect of emphasizing or de-emphasizing different elements of the text, creating temporal-textual emphases, which can result from score indications and performer decisions alike. In both instances, performances have unique temporal-textual emphases, which are places where a performer's use of time emphasizes or deemphasizes particular words or musical phrases.

Individually, these surface-level emphases highlight individual words or phrases, but when their emphasis crosses another one in a higher (or deeper) structural level, a structural crossing links the two words or phrases. These crossings, which occur in the mid-ground and background of tempo hierarchies, are evident by the intersections of color extensions from the graph's surface level. Analysis of structural crossings can reveal the unique timing structure each performer imposes on the text and what it communicates, thus providing fresh and nuanced insight into a performer's interpretation of a text. This insight can form the basis of a hypothesis about a performer's mindset about a particular aria or character, which in turn points towards the use of this kind of analysis for better understanding the creative process. Confirming such hypotheses via interview, as in the present study, or other secondary sources makes this capability useful for interrogating the creative process, potentially even in the absence of any supporting extra-recording evidence.

\subsection{Statistical and Network analysis}

Performances of the same work share an inherent connectedness. Because performance is a human behavior with measurable physical properties in and across time, it is readily analyzable through time-series analysis. Furthermore, opera is a genre whose performances are inextricable from notions of tradition and convention. Tradition is a kind of perceptual repository that connects events across time by reflecting events that have occurred, allowing new events to enter the pool experience, and reflects change in the overall perception. Convention invokes a sense of authority or expectation. A recent definition of tradition delineates this difference, and stipulates that a tradition can become a convention if someone uses it as a benchmark of acceptable practice (emphasis added). Semantic analysis of definitions of statistical means and tradition suggests that both behave nearly identically for data and behaviors. [9]

The first step in establishing embodiments a tradition for "In questa Reggia" is the calculation of chronologically progressive averages of tempo data for each excerpt, which reflects tradition's nature as passive and perceptual, and the data of which can help construct Time Scape visualizations of points along a tradition's evolution. Thus, the earliest numerical representation of a tradition (A1-2 in Figure 3) is the numerical average of the data streams for the first two performances with respect to chronology $(\mathrm{P} 1, \mathrm{P} 2)$. The second point in the tradition $(\mathrm{A} 1-3)$ is the average of the first three performances, and the most recent (A1-19) is the average of all performance tempo data sets.

As Cook and Sapp showed in their forensic application to performances attributed to Joyce Hatto, Pearson correlation is one means of measuring the strength of similarity between sets of linear data [3]. $R$-values for the current set of performances (P1-P19) and traditions (A1-2-A1-19) characterize interactivity between these 38 entities. Single Pearson correlation values can only describe the total similarity of two data sets, rather than any constituent parts of them. Parsing the data sets into sub sequences thus is necessary for a fuller measure of similarity. Opera arias of the late $19^{\text {th }}$ and early $20^{\text {th }}$ centuries can be difficult to subdivide. Developing apparatuses for automating other subdivision possibilities, including formal divisions of text and music and notated instructions, is an aim of future research. 
Cytoscape allows plotting and analyzing the network of performances and points in the tradition is possible [4].

\section{PERFORMANCE PROFILING}

An example from Turandot's performance history highlights the focus on textual emphasis of my adaptations of Time Scape analyses. ${ }^{1}$ This analysis reveals different textualtemporal emphases, which, emphasize each performance the unique identity of each performance and insinuate interpretation.

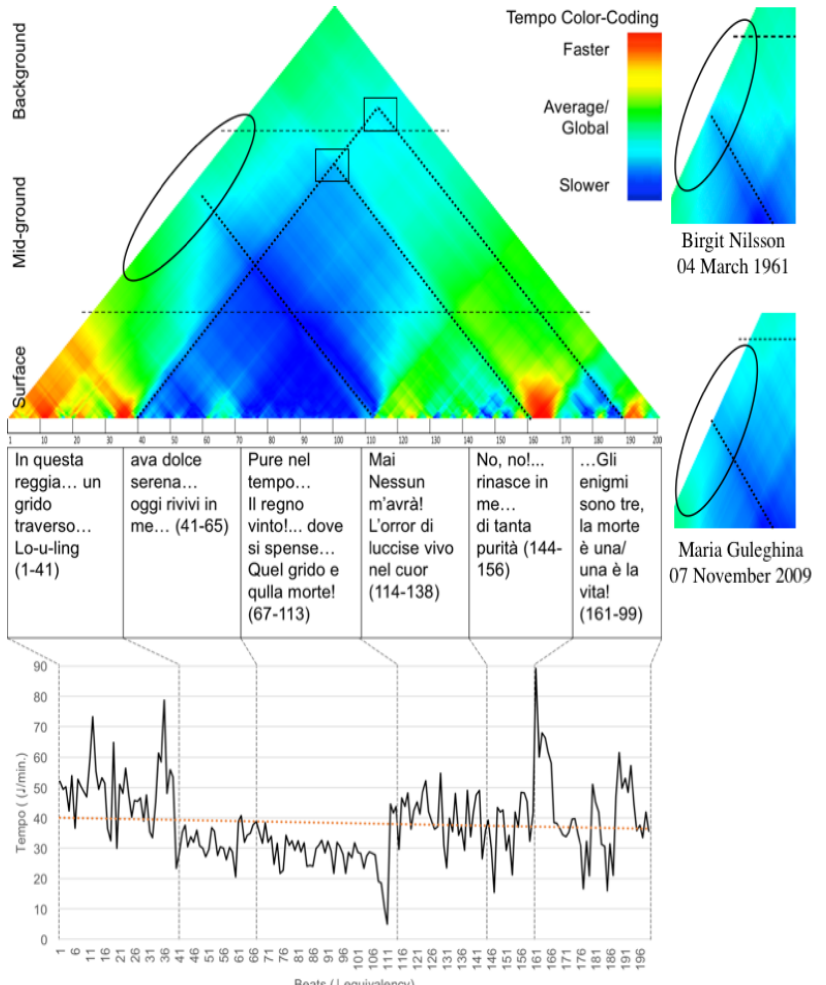

Figure 1. Time Scape, text, and tempo contour graph for Andréa Gruber 29 January 2005 "In questa Reggia."

Excerpts of Time Scapes for Birgit Nilsson 04 March 1961 and Maria Guleghina 07 November 2009.

Time Scape analysis of opera performance is a useful means of establishing how individual performers structure the temporal aspects of their interpretations. Tempo analysis in relationship to an aria's text-temporal-textual emphasis-is particularly appropriate for opera, which relies upon musical-dramatic pacing to convey its narrative. Its usefulness for the works of Giacomo Puccini, who was notoriously finicky about tempi for his works, is natural. Perhaps the greatest significance of this approach emerges in combination with primary resources from performers.

A Time Scape representing Andréa Gruber's 29 January 2005 performance appears in Figure 1. Much of the tempo hierarchy in Gruber's performance is similar to the other eighteen performances of this aria in the current study. Most striking about Gruber's temporal hierarchy is that, unlike for most other performances of "In questa Reggia" at the Met, the higher-level structural feature resulting from her tempi across beats 41-113 does not extend to the left edge of the graph. This feature results from Gruber's tempi through the aria's first two formal sections (b. 1-41 and 41-113), which temporally emphasize Lou-o-Ling's scream and death to lesser degrees than performances where this feature extends to the graph's edge, which the excerpted images from Time Scapes for performances by Birgit Nilsson performance in 1961 and Maria Guleghina in 2009 show in Figure 2. This feature, combined with structural crossings (boxed) between it and other surface level events (lines) suggests

\footnotetext{
${ }^{1}$ Time Scapes for other performances are available upon request.
}

a temporal-textual structure in which Gruber's Turandot is not entirely consumed or motivated by quest for revenge, and that Calaf's arrival represents a possible rescue from her circumstances. Gruber subsequently confirmed my assessment of her mindset for Turandot in this aria in an interview; for her, Calaf can break the chains of Turandot's captivity in her father's game of suitors, riddles, and beheadings by satisfying the riddles and arousing her erotic love.

\section{CORRELATION NETWORK ANALYSIS}

\subsection{Correlation}

Because Pearson correlation is most effective with linear data, segmentation of data for "In questa Reggia" into its three tempo sections provides a more accurate view of connections amidst performances and points in their resulting tradition. can assess the strength of relationships between sets of data than measuring correlation for global tempo alone. Focusing on first and second order correlations reveals the strongest clusters and suggests a perhaps unexpected influence on the evolution of this aria's performance history at the Metropolitan Opera.

In this data set, correlation values between points in the tradition (e.g. - A1-2 $\rightarrow$ A1-3) are generally significantly stronger than those between any two performances and those between performances and points in the tradition, and the tradition becomes more similar to itself as it results from more performances. This trait is not surprising from a data analysis standpoint, and it emphasizes that tradition, as a perceptual concept, retains awareness of previous events, thereby smoothing out minutiae of individual performances as results from, rather than influences them. [9] However, as Figure 3 shows, performances sometimes relate most strongly to each other; which stresses that understanding the reasons for such occurrences-modeling, whether deliberate or unconscious, homage, or simple coincidence-merits further consideration.

\subsection{Network}

\subsubsection{Relationships of performances and points in a tradition}

Cytoscape's circle attribute (top 2) and orthogonal layouts (bottom) for each of the three tempo subsections of "In questa Reggia" produce Figure 2's individual graphs. ${ }^{2}$ This network's nodes each represent a performance $(\mathrm{P} 1, \mathrm{P} 2$, etc.) and points in the tradition's evolution (A1-2, A1-3, etc.) both numbered to reflect chronological ordering. Edges reflect the Pearson correlation values between nodes, with darker edges representing higher $R$-values. The first and second order correlation network appearing as Figure 3 shows the two strongest similarities from each performance $(\mathrm{P})$ and point in the tradition (A). Points in the tradition are targets more often than performances, emphasizing tradition's intermedial nature.

Every point in the tradition's evolution is a first or second order correlation source node for the point in the tradition immediately following it, except A1-4, which targets A1-3 in a third order correlation (not shown in Figure 3). Most of the points in the tradition also connect at a first or second correlation order to their respective immediate predecessors, reflecting a reflexivity that aligns to conceptions of tradition's connectivity, giving further viability to data-based modelling and analysis of tradition.

\subsubsection{Clusters of production practice}

Two distinct groupings in each tempo section emerge, with aligning to one of the two productions in the recorded corpus of Turandot at the Met. Each section of the aria contains outlier performances or points in the tradition's evolution. For the aria's

\footnotetext{
${ }^{2}$ http://www.cytoscape.org/
} 
introductory section - at the top of Figure 2-P5 (Marion Lippert), P7 (Elinor Ross), and P8 (Ingrid Bjoner) each aligns most similarly to performances or points in the tradition associated with the Franco Zeffirelli production. Only Ghena Dimitrova's 1996 performance (P15) relates most strongly to earlier events in the performance history. In the $4 / 8$ and $2 / 8$ section of the aria, only P5 and P7 continue to relate most strongly to events associated with the production not in use at the time of their performance. For the aria's conclusion, all performances relate most strongly to events in their associated tradition. A1-9 and A1-10 are exceptions, but they have only comparatively weak connections to the performances at the end of the Beaton production and P3 (Birgit Nilsson) connects to A119. Even with such cross-connections, the clear distinction between tempo data, the evolving tradition, and Turandot's production history. Each case reveals possibilities of influence and suggests how conventional modes of musicological inquiry could benefit by the data suggest focused research parameters.
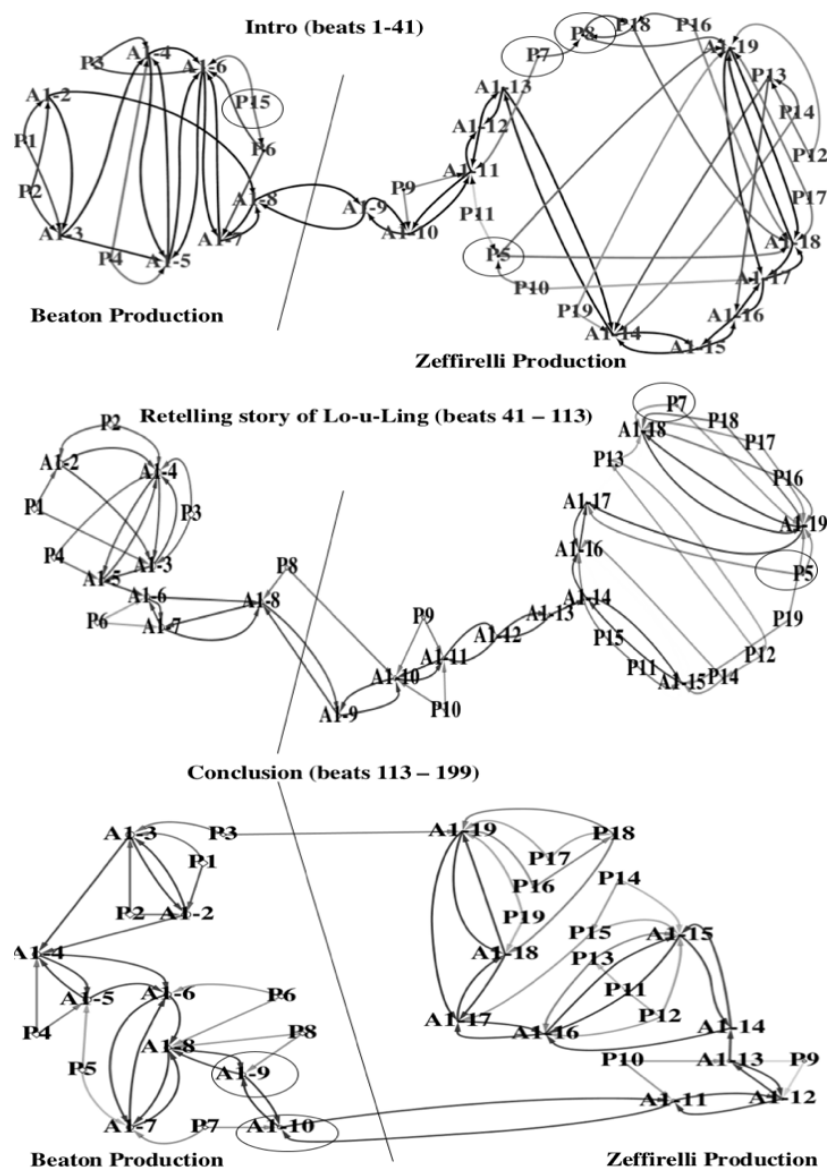

Figure 2. Network clusters for each of three sections in "In questa Reggia." "P" nodes represent performances; "A" nodes represent points in the tradition. Edges reflect Pearson correlation values (darker is stronger).

\subsubsection{Betweenness centrality and cultural significance}

Betweenness centrality reflects the degree to which a node is a bridge along the shortest path between any two other nodes, reflecting a node's intermedial significance in a network because it. A1-11 and P13 have the highest degrees of betweenness centrality for the introduction, while A1-10 and P14 and A1-10 and P12 have the highest betweenness centrality factors for the second and third sections, respectively. Aside from A1-12, all other high betweenness centrality nodes result from performances led by Nello Santi, who is the most frequent conductor of Turandot in the available corpus of recordings. Betweenness centrality confirms Santi's significance on the particular tempo practices in "In questa Reggia" and points to the consistency with which he shaped performances during his tenure at the Metropolitan Opera.

\section{CONCLUSIONS AND FUTURE WORK}

The methods I have demonstrated in this paper are useful for examining the creative process of individual performances and how they evolve and interrelate over the course of a performance history. Furthermore, the use of a statistical mean as a model for a tradition mirrors tradition's additive nature, especially as it relates to perception. Situating both performances and embodiments of tradition in a network allows the analysis of interrelationships, demonstrates a clear coalescence around production history, and confirms the prominence of Turandot's most common conductor - in appearances and musical practices.

This research is a step toward conceptual inquiry of tradition, specifically how its role in operatic production and consumption is similar to and different from convention. The current paper asserts tradition's role as an intermedial and perceived repository, leaving convention as, among other things, a tradition invoked as an aesthetic model to follow. Future work based on the methods in this project will continue to fill knowledge gaps while refining analysis of the creative process.

\section{ACKNOWLEDGMENTS}

The author acknowledges the support of the New York Public Library's Rodgers and Hammerstein Archive of Recorded Sound and the Metropolitan Opera for access to the recordings and production history sources. This work has been supported by the University of Florida Center for European Studies and the University of Florida Graduate School.

\section{REFERENCES}

[1] Cannam, Chris, Christian Landone, and Mark Sandler. Sonic Visualiser. 2010. http://www.sonicvisualiser.org/.

[2] Cook, Nicholas. Beyond the Score: Music as Performance. Oxford, Oxford University Press, 2013.

[3] Cook, Nicholas, and Craig Sapp, "Purely coincidental? Joyce Hatto and Chopin's Mazurkas," http://www.charm.rhul.ac.uk/projects/p2_3_2.html.

[4] Cytoscape 3 User Manual. Last modified on 25 March 2016. http://wiki.cytoscape.org/ Cytoscape_3/UserManual.

[5] Fabian, Dorottya, Renee Timmers, and Emery Schubert. Expressiveness in Music Performance: Empirical Approaches Across Styles and Cultures. Oxford, Oxford University Press, 2014.

[6] Gruber, Andréa. Interview by the author. Personal Interview. New York, 29 January 2015.

[7] Johnson, Victoria, Jane F. Fulcher, and Thomas Ertman. Opera and Society in Italy and France from Monteverdi to Bourdieu. Cambridge: Cambridge University Press, 2007.

[8] Mazurka Project. Last modified on August 21, 2006. http://www.mazurka.org.uk/ana/timescape/.

[9] Neumann, Joshua. Toward Defining Tradition: A Statistical and Network Analysis of Giacomo Puccini's Turandot at the Metropolitan Opera. PhD diss., University of Florida, 2016.

[10] Puccini, Giacomo, Giuseppe Adami, Renato Simoni, and Franco Alfano. Turandot. Milano: G. Ricordi, 1926.

[11] Ricci, Luigi. Puccini interprete di se stesso. Milan: Ricordi, 1954.

[12] Tools Developed by the CHARM Mazurka Project. Last modified 29 October 2015. http://www.charm.rhul.ac.uk/analysing/p9_4.html. 